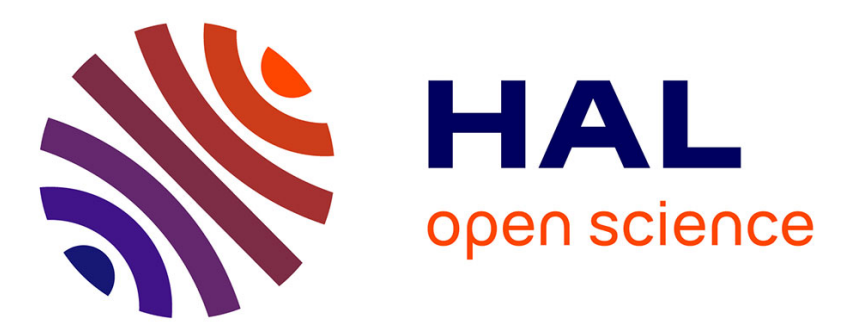

\title{
Two-generator numerical semigroups and Fermat and Marsenne numbers
}

Shalom Eliahou, Jorge Ramirez Alfonsin

\section{To cite this version:}

Shalom Eliahou, Jorge Ramirez Alfonsin. Two-generator numerical semigroups and Fermat and Marsenne numbers. SIAM Journal on Discrete Mathematics, 2011, 25, pp.622-630. $10.1137 / 100787283$. hal-00812743

\section{HAL Id: hal-00812743 \\ https://hal.science/hal-00812743}

Submitted on 22 Apr 2013

HAL is a multi-disciplinary open access archive for the deposit and dissemination of scientific research documents, whether they are published or not. The documents may come from teaching and research institutions in France or abroad, or from public or private research centers.
L'archive ouverte pluridisciplinaire HAL, est destinée au dépôt et à la diffusion de documents scientifiques de niveau recherche, publiés ou non, émanant des établissements d'enseignement et de recherche français ou étrangers, des laboratoires publics ou privés. 


\title{
Two-generator numerical semigroups and Fermat and Mersenne numbers
}

\author{
Shalom Eliahou and Jorge Ramírez Alfonsín
}

\begin{abstract}
Given $g \in \mathbb{N}$, what is the number of numerical semigroups $S=$ $\langle a, b\rangle$ in $\mathbb{N}$ of genus $|\mathbb{N} \backslash S|=g$ ? After settling the case $g=2^{k}$ for all $k$, we show that attempting to extend the result to $g=p^{k}$ for all odd primes $p$ is linked, quite surprisingly, to the factorization of Fermat and Mersenne numbers.
\end{abstract}

Keywords: Semigroups, factorization, Fermat, Mersenne.

MSC 2010: 05A15, 11A05, 11A51, 20M14

\section{Introduction}

A numerical semigroup is a subset $S$ of $\mathbb{N}$ containing 0 , stable under addition, and with finite complement $G(S)=\mathbb{N} \backslash S$. The elements of $G(S)$ are called the gaps of $S$, and their number is denoted $g(S)$ and called the genus of $S$. The Frobenius number of $S$ is its largest gap. See [6] for more details. If $a_{1}, \ldots, a_{r}$ are positive integers with $\operatorname{gcd}\left(a_{1}, \ldots, a_{r}\right)=1$, then they generate a numerical semigroup $S=\mathbb{N} a_{1}+\cdots+\mathbb{N} a_{r}$, denoted $S=\left\langle a_{1}, \ldots, a_{r}\right\rangle$. For example, the numerical semigroup $S=\langle 4,5,7\rangle$ has gaps $G(S)=\{1,2,3,6\}$, whence genus $g(S)=4$ and Frobenius number 6 . It is well known that every numerical semigroup admits a unique finite minimal generating set [3].

Given $g \in \mathbb{N}$, what is the number $n_{g}$ of numerical semigroups $S$ of genus $g$ ? Maria Bras-Amorós recently determined $n_{g}$ for all $g \leq 50$ by computer. On this basis, she made three conjectures suggesting that the numbers $n_{g}$ behave closely like the Fibonacci numbers $[1,2]$. For instance, the inequality $n_{g} \geq n_{g-1}+n_{g-2}$, valid for $g \leq 50$, is conjectured to hold for all $g$. 
We propose here the refined problem of counting numerical semigroups $S$ of genus $g$ with a specified number of generators.

Notation 1.1 Given $g, r \geq 1$, let $n(g, r)$ denote the number of numerical semigroups $S$ of genus $g$ having a minimal generating set of cardinality $r$.

Of course $n_{g}=\sum_{r \geq 1} n(g, r)$. Is there an explicit formula for $n(g, r)$, and might it be true that $n(g, r) \geq n(g-1, r)+n(g-2, r)$ ?

In this paper, we focus on the case $r=2$, i.e. on numerical semigroups $S=\langle a, b\rangle$ with $\operatorname{gcd}(a, b)=1$. The genus of $S$ is equal to $(a-1)(b-1) / 2$, by a classical theorem of Sylvester. This allows us to show in Section 2 that $n(g, 2)$ depends on the factorizations of both $2 g$ and $2 g-1$, and to determine $n(g, 2)$ when $2 g-1$ is prime. In Section 3 , we determine $n(g, 2)$ for $g=2^{k}$ and all $k \geq 1$. We then tackle the case $g=p^{k}$ for odd primes $p$ in Section 4 . On the one hand, we provide explicit formulas for $n\left(p^{k}, 2\right)$ when $k \leq 6$. On the other hand, we show that obtaining similar formulas for all $k \geq 1$ is linked to the factorization of Fermat and Mersenne numbers. We conclude with a few open questions about $n(g, 2)$.

\section{Basic properties of $n(g, 2)$}

We will show that $n(g, 2)$ is linked with the factorizations of $2 g$ and $2 g-1$. For this, we need the following theorem of Sylvester [7].

Theorem 2.1 Let $a, b$ be coprime positive integers, and let $S=\langle a, b\rangle$. Then $\max G(S)=a b-a-b$, and for all $x \in\{0,1, \ldots, a b-a-b\}$, one has

$$
x \in G(S) \Longleftrightarrow a b-a-b-x \in S .
$$

In particular, $g(S)=(a-1)(b-1) / 2$.

\subsection{Link with factorizations of $2 g$ and $2 g-1$}

We first derive that $n(g, 2)$ is the counting function of certain particular factorizations of $2 g$. As usual, the cardinality of a set $X$ will be denoted $|X|$.

Proposition 2.2 Let $g \geq 1$ be a positive integer. Then we have

$$
n(g, 2)=\left|\left\{(u, v) \in \mathbb{N}^{2} \mid 1 \leq u \leq v, u v=2 g, \operatorname{gcd}(u+1, v+1)=1\right\}\right| .
$$


Proof. Indeed, let $S=\langle a, b\rangle$ with $1 \leq a \leq b$ and $\operatorname{gcd}(a, b)=1$, and assume that $g(S)=g$. By Theorem 2.1, we have $g=(a-1)(b-1) / 2$, i.e. $2 g=(a-1)(b-1)$. The claim follows by setting $u=a-1, v=b-1$.

A first consequence is that every $g \geq 1$ is the genus of an appropriate 2-generator numerical semigroup.

Corollary $2.3 n(g, 2) \geq 1$ for all $g \geq 1$.

Proof. This follows from the factorization $2 g=u v$ with $u=1, v=2 g$. Concretely, the numerical semigroup $S=\langle 2,2 g+1\rangle$ has genus $g$.

Our next remark shows that $n(g, 2)$ is also linked with the factors of $2 g-1$.

Lemma 2.4 Let $g \geq 1$ be a positive integer, and let $2 g=u v$ with $u, v$ positive integers. Then $\operatorname{gcd}(u+1, v+1)$ divides $2 g-1$.

Proof. Set $\delta=\operatorname{gcd}(u+1, v+1)$. Then $u \equiv v \equiv-1 \bmod \delta$, and therefore $2 g=u v \equiv 1 \bmod \delta$.

\subsection{The case where $2 g-1$ is prime}

We can now determine $n(g, 2)$ when $2 g-1$ is prime. As customary, for $n \in \mathbb{N}$ we denote by $d(n)$ the number of divisors of $n$ in $\mathbb{N}$.

Proposition 2.5 Let $g \geq 3$, and assume that $2 g-1$ is prime. Then

$$
n(g, 2)=d(2 g) / 2 \text {. }
$$

In particular, $n(g, 2)=d(g)$ if $g$ is odd.

Proof. Let $2 g=u v$ be any factorization of $2 g$ in $\mathbb{N}$. We claim that $\operatorname{gcd}(u+1, v+1)=1$. Indeed, by Lemma 2.4 we know that $\operatorname{gcd}(u+1, v+1)$ divides $2 g-1$. Assume for a contradiction that $\operatorname{gcd}(u+1, v+1) \neq 1$. Then $\operatorname{gcd}(u+1, v+1)=2 g-1$, since $2 g-1$ is assumed to be prime. It follows that $u, v \geq 2 g-2$, implying

$$
2 g=u v \geq 4(g-1)^{2} .
$$


However, the inequality $2 g \geq 4(g-1)^{2}$, while true at $g=2$, definitely fails for $g \geq 3$ as assumed here. Thus $\operatorname{gcd}(u+1, v+1)=1$, as claimed. Hence, by Proposition 2.2, we have

$$
n(g, 2)=\left|\left\{(u, v) \in \mathbb{N}^{2} \mid u \leq v, 2 g=u v\right\}\right| .
$$

Clearly $2 g$ counts as many divisors $u<\sqrt{2 g}$ as divisors $v>\sqrt{2 g}$. Moreover $2 g$ is not a perfect square. This is clear for $g=3$ or 4 . If $g \geq 5$ and $2 g=a^{2}$ with $a \in \mathbb{N}$, then $a \geq 3$ and $2 g-1=a^{2}-1=(a-1)(a+1)$, contradicting the primality of $2 g-1$. We conclude from $(1)$ that $n(g, 2)=d(2 g) / 2$. Finally, if $g$ is further assumed to be odd, then clearly $d(2 g) / 2=d(g)$.

Proposition 2.5 cannot be extended to $g=2$, even though $2 g-1$ is prime. Indeed $n(2,2)=1$ as easily seen, whereas $d(4) / 2$ is not even an integer.

Since $n(g, 2)$ is controlled by the factorizations of both $2 g$ and $2 g-1$, its determination is expected to be hard in general, even if the factors of $g$ are known. Nevertheless, below we determine $n(g, 2)$ when $g=2^{k}$ for all $k \in \mathbb{N}$, despite the fact that the prime factors of $2^{k+1}-1$ are generally unknown.

\section{The case $g=2^{k}$}

Let $g=2^{p-1}$ with $p$ an odd prime, and assume that $2 g-1$ is prime. ${ }^{1}$ Proposition 2.5 then applies, and gives

$$
n\left(2^{p-1}, 2\right)=d\left(2^{p}\right) / 2=(p+1) / 2 .
$$

But we shall now determine $n\left(2^{k}, 2\right)$ for all $k \in \mathbb{N}$, and show that its value only depends on the largest odd factor $s$ of $k+1$.

Theorem 3.1 Let $g=2^{k}$ with $k \in \mathbb{N}$. Write $k+1=2^{\mu} s$ with $\mu \in \mathbb{N}$ and $s$ odd. Then

$$
n\left(2^{k}, 2\right)=(s+1) / 2 .
$$

Proof. Since $2 g=2^{k+1}$, the only integer factorizations $2 g=u v$ with $1 \leq u \leq v$ are given by

$$
u=2^{i}, v=2^{k+1-i}
$$

\footnotetext{
${ }^{1}$ In fact a Mersenne prime, since $2 g-1=2^{p}-1$. See also Section 4.2.
} 
with $0 \leq i \leq(k+1) / 2$. In order to determine $n\left(2^{k}, 2\right)$ with Proposition 2.2, we must count those $i$ in this range for which $\operatorname{gcd}\left(2^{i}+1,2^{k+1-i}+1\right)=1$. This condition is taken care of by the following claim.

Claim. We have $\operatorname{gcd}\left(2^{i}+1,2^{k+1-i}+1\right)=1$ if and only if $2^{\mu}$ divides $i$.

The claim is proved by examining separately the cases where $2^{\mu}$ divides $i$ or not.

- Case 1: $2^{\mu}$ divides $i$. Assume for a contradiction that there is a prime $p$ dividing $\operatorname{gcd}\left(2^{i}+1,2^{k+1-i}+1\right)$. Then $p$ is odd, and we have

$$
2^{i} \equiv 2^{k+1-i} \equiv-1 \bmod p
$$

It follows that

$$
2^{2 i} \equiv 2^{k+1} \equiv 1 \bmod p .
$$

Let $m$ denote the multiplicative order of $2 \bmod p$. It follows from (3) that $m$ divides $\operatorname{gcd}(2 i, k+1)$. Now, in the present case, we have

$$
\operatorname{gcd}(2 i, k+1)=\operatorname{gcd}(i, k+1),
$$

since $2^{\mu}$ divides $i$ and $k+1$, while $2^{\mu+1}$ divides $2 i$ without dividing $k+1$. Consequently $m$ divides $i$, not only $2 i$. Hence $2^{i} \equiv 1 \bmod p$, in contradiction with (2). Therefore $\operatorname{gcd}\left(2^{i}+1,2^{k+1-i}+1\right)=1$, as desired.

- Case 2: $2^{\mu}$ does not divide $i$. We may then write $i=2^{\nu} j$ with $j$ odd and $\nu<\mu$. Set $q=2^{2^{\nu}}+1$, and note that $q \geq 3$. We claim that $q$ divides $\operatorname{gcd}\left(2^{i}+1,2^{k+1-i}+1\right)$. Indeed, observe that

$$
2^{2^{\nu}} \equiv-1 \bmod q
$$

by definition of $q$. Since $i=2^{\nu} j$ with $j$ odd, we have

$$
2^{i}+1=\left(2^{2^{\nu}}\right)^{j}+1 \equiv(-1)^{j}+1 \equiv 0 \bmod q .
$$

Similarly, we have $k+1-i=2^{\nu} j^{\prime}$ where $j^{\prime}=2^{\mu-\nu} s-j$. Then $j^{\prime}$ is odd, since $\mu-\nu>0$ and $j$ is odd. As above, this implies that

$$
2^{k+1-i}=\left(2^{2^{\nu}}\right)^{j^{\prime}}+1 \equiv 0 \bmod q .
$$

It follows that $q$ divides $\operatorname{gcd}\left(2^{i}+1,2^{k+1-i}+1\right)$, thereby settling the claim. 
We may now conclude the proof. Indeed, the above claim yields

$$
\begin{aligned}
n\left(2^{k}, 2\right) & =\left|\left\{i \mid 0 \leq i \leq(k+1) / 2, i \equiv 0 \bmod 2^{\mu}\right\}\right| \\
& =\left|\left\{j \mid 0 \leq j \leq(k+1) / 2^{\mu+1}\right\}\right| \\
& =\left\lfloor(k+1) / 2^{\mu+1}+1\right\rfloor=(s+1) / 2 .
\end{aligned}
$$

Corollary 3.2 For every $N \geq 1$, there are infinitely many $g \geq 1$ such that $n(g, 2)=N$.

Proof. Let $s=2 N-1$. Then $s$ is odd, and for all $k=2^{\mu} s-1$ with $\mu \in \mathbb{N}$, we have $n\left(2^{k}, 2\right)=(s+1) / 2=N$ by Theorem 3.1 .

In particular, there are infinitely many $g \geq 1$ for which $n(g, 2)=1$. Since $n(h, 2) \geq 1$ for all $h \geq 1$, the inequality $n(g, 2) \geq n(g-1,2)+n(g-2,2)$ fails to hold infinitely often. This says nothing, of course, about the original conjecture $n_{g} \geq n_{g-1}+n_{g-2}$ of Bras-Amorós.

\section{The case $g=p^{k}$ for odd primes $p$}

We have determined $n\left(2^{k}, 2\right)$ for all $k \geq 1$. Attempting to similarly determine $n\left(p^{k}, 2\right)$ for odd primes $p$ leads to a somewhat paradoxical situation. Indeed, while the case where $k$ is small is relatively straightforward, formidable difficulties arise when $k$ grows. This Jekyll-and-Hyde behavior is shown below.

\subsection{When $k$ is small}

Given positive integers $q_{1}, \ldots, q_{t}$, we denote by

$$
\rho_{q_{1}, \ldots, q_{t}}: \mathbb{Z} \rightarrow \mathbb{Z} / q_{1} \mathbb{Z} \times \cdots \times \mathbb{Z} / q_{t} \mathbb{Z}
$$

the canonical reduction morphism $\rho_{q_{1}, \ldots, q_{t}}(n)=\left(n \bmod q_{1}, \ldots, n \bmod q_{t}\right)$, and shall write $n \equiv \neg a \bmod q$ instead of $n \not \equiv a \bmod q$. For example, the condition

$$
\rho_{3,5,17}(p)=(2, \neg 3, \neg 8)
$$

means that $p \equiv 2 \bmod 3, p \not \equiv 3 \bmod 5$ and $p \not \equiv 8 \bmod 17$. 
Proposition 4.1 Let $p$ be an odd prime number. Then we have:

$$
\begin{aligned}
& \text { 1. } n(p, 2)=\left\{\begin{array}{lll}
1 & \text { if } & \rho_{3}(p)=2 \\
2 & \text { if } & \rho_{3}(p)=\neg 2,
\end{array}\right. \\
& \text { 2. } n\left(p^{2}, 2\right)=3, \\
& \text { 3. } n\left(p^{3}, 2\right)=\left\{\begin{array}{lll}
1 & \text { if } & \rho_{3,5}(p)=(2,2) \\
2 & \text { if } & \rho_{3,5}(p)=(2, \neg 2) \\
3 & \text { if } & \rho_{3,5}(p)=(\neg 2,2) \\
4 & \text { if } & \rho_{3,5}(p)=(\neg 2, \neg 2),
\end{array}\right. \\
& \text { 4. } n\left(p^{4}, 2\right)=\left\{\begin{array}{lll}
4 & \text { if } & \rho_{7}(p)=3 \\
5 & \text { if } & \rho_{7}(p)=\neg 3,
\end{array}\right. \\
& \text { 5. } n\left(p^{5}, 2\right)=\left\{\begin{array}{lll}
1 & \text { if } & \rho_{3,5,17}(p)=(2,3,8) \\
2 & \text { if } & \rho_{3,5,17}(p)=(2,3, \neg 8) \text { or }(2, \neg 3,8) \\
3 & \text { if } & \rho_{3,5,17}(p)=(2, \neg 3, \neg 8) \\
4 & \text { if } & \rho_{3,5,17}(p)=(\neg 2,3,8) \\
5 & \text { if } & \rho_{3,5,17}(p)=(\neg 2,3, \neg 8) \text { or }(\neg 2, \neg 3,8) \\
6 & \text { if } & \rho_{3,5,17}(p)=(\neg 2, \neg 3, \neg 8),
\end{array}\right. \\
& \text { 6. } n\left(p^{6}, 2\right)=\left\{\begin{array}{lll}
6 & \text { if } & \rho_{31}(p)=15 \\
7 & \text { if } & \rho_{31}(p)=\neg 15 .
\end{array}\right.
\end{aligned}
$$

With the Chinese Remainder Theorem, the above result implies that $n\left(p^{3}, 2\right)$ depends on the class of $p$ modulo 15 , and that $n\left(p^{4}, 2\right), n\left(p^{5}, 2\right)$ and $n\left(p^{6}, 2\right)$ depend on the class of $p$ modulo 7, 255 and 31, respectively.

Proof. Let $k \leq 6$. We determine $n\left(p^{k}, 2\right)$ using Proposition 2.2. As $p$ is an odd prime, counting the factorizations $2 p^{k}=u v$ with $u \leq v$ and $\operatorname{gcd}(u+1, v+1)=1$ amounts to count the number of exponents $i$ in the range $0 \leq i \leq k$ satisfying the condition

$$
\operatorname{gcd}\left(p^{i}+1,2 p^{k-i}+1\right)=1 .
$$

A convenient way to ease the computation of this gcd is to replace $p$ by a variable $x$ and to reduce, in the polynomial ring $\mathbb{Z}[x]$, the greatest common divisor of $x^{i}+1$ and $2 x^{j}+1$ to the simpler form

$$
\operatorname{gcd}\left(x^{i}+1,2 x^{j}+1\right)=\operatorname{gcd}(f, g),
$$


where either polynomial $f$ or $g$ is constant. Polynomials are used here precisely for allowing such degree considerations. Since $\operatorname{gcd}\left(p^{i}+1,2 p^{j}+1\right)$ is odd, we may equivalently work in the rings $\mathbb{Z}\left[2^{-1}\right]$ or $\mathbb{Z}\left[2^{-1}, x\right]$, where 2 is made invertible. Note that these rings are still unique factorization domains.

We obtain the following table, with a method explained below. For simplicity, we write $(f, g)$ rather than $\operatorname{gcd}(f, g)$, and 1 whenever either $f$ or $g$ is invertible in the ring $\mathbb{Z}\left[2^{-1}, x\right]$. The cases $i=0$ and $j=0$ are not included, since then $x^{i}+1$ and $2 x^{j}+1$ are already constant, respectively.

\begin{tabular}{c||c|c|c|c|c|c|} 
gcd & $2 x+1$ & $2 x^{2}+1$ & $2 x^{3}+1$ & $2 x^{4}+1$ & $2 x^{5}+1$ & $2 x^{6}+1$ \\
\hline \hline$x+1$ & 1 & $(x+1,3)$ & 1 & $(x+1,3)$ & 1 & $(x+1,3)$ \\
\hline$x^{2}+1$ & $(2 x+1,5)$ & 1 & $(2 x-1,5)$ & $\left(x^{2}+1,3\right)$ & $(2 x+1,5)$ & 1 \\
\hline$x^{3}+1$ & $(2 x+1,7)$ & $(x-2,9)$ & 1 & $(2 x-1,9)$ & $(x+2,7)$ & $\left(x^{3}+1,3\right)$ \\
\hline$x^{4}+1$ & $(2 x+1,17)$ & $\left(2 x^{2}+1,5\right)$ & $(x-2,17)$ & 1 & $(2 x-1,17)$ & $\left(2 x^{2}-1,5\right)$ \\
\hline$x^{5}+1$ & $(2 x+1,31)$ & $(x+4,33)$ & $(4 x+1,31)$ & $(x-2,33)$ & 1 & $(2 x-1,33)$ \\
\hline$x^{6}+1$ & $(2 x+1,65)$ & $\left(2 x^{2}+1,7\right)$ & $\left(2 x^{3}+1,5\right)$ & $\left(x^{2}-2,9\right)$ & $(x-2,65)$ & 1 \\
\hline
\end{tabular}

Table 1: Reduction of $\operatorname{gcd}\left(x^{i}+1,2 x^{j}+1\right)$ for $1 \leq i, j \leq 6$.

In order to construct this table, we use the most basic trick for computing gcd's in a unique factorization domain $A$, namely:

$$
g_{1} \equiv g_{2} \bmod f \Rightarrow \operatorname{gcd}\left(f, g_{1}\right)=\operatorname{gcd}\left(f, g_{2}\right)
$$

for all $f, g_{1}, g_{2} \in A$. As an illustration, let us reduce $\operatorname{gcd}\left(x^{2}+1,2 x^{3}+1\right)$ to the form (4) in the ring $\mathbb{Z}\left[2^{-1}, x\right]$. We have

$$
\begin{aligned}
\operatorname{gcd}\left(x^{2}+1,2 x^{3}+1\right) & =\operatorname{gcd}\left(x^{2}+1,-2 x+1\right) \\
& =\operatorname{gcd}\left(2^{-2}+1,-2 x+1\right) \\
& =\operatorname{gcd}\left(1+2^{2}, 2 x-1\right),
\end{aligned}
$$

where steps (6) and (7) follow from (5) and the respective congruences

$$
\begin{aligned}
& x^{2} \equiv-1 \bmod \left(x^{2}+1\right), \\
& x \equiv 2^{-1} \bmod (-2 x+1) .
\end{aligned}
$$

Hence $\operatorname{gcd}\left(x^{2}+1,2 x^{3}+1\right)=\operatorname{gcd}(2 x-1,5)$, as displayed in Table 4.1. 
Now, from that table, it is straightforward to determine those pairs of exponents $i, j$ with $i+j \leq 6$ and those odd primes $p$ for which

$$
\operatorname{gcd}\left(p^{i}+1,2 p^{j}+1\right)=1,
$$

and hence to obtain the stated formulas for $n\left(p^{k}, 2\right)$. Consider, for instance, the case $k=3$. We shall count those exponents $i \in\{0,1,2,3\}$ for which

$$
\operatorname{gcd}\left(p^{i}+1,2 p^{3-i}+1\right)=1 \text {. }
$$

$\underline{i=0}$ : Condition (9) is always satisfied.

$\underline{i=1}$ : Table 4.1 gives $\operatorname{gcd}\left(p+1,2 p^{2}+1\right)=\operatorname{gcd}(p+1,3)$, which equals 1 exactly when $p \not \equiv 2 \bmod 3$.

$\underline{i=2}$ : Table 4.1 gives $\operatorname{gcd}\left(p^{2}+1,2 p+1\right)=\operatorname{gcd}(2 p+1,5)$, which equals 1 exactly when $p \not \equiv 2 \bmod 5$.

$\underline{i=3}$ : Finally, we have $\operatorname{gcd}\left(p^{3}+1,3\right)=1$ exactly when $p \not \equiv 2 \bmod 3$.

It follows that $n\left(p^{3}, 2\right)$ is entirely determined by the classes of $p \bmod 3$ and 5 , with a value ranging from 1 to 4 depending on whether $\rho_{3,5}(p)$ equals $(2,2),(2, \neg 2),(\neg 2,2)$ or $(\neg 2, \neg 2)$, as stated.

The cases $k=1,2,4,5,6$ are similar and left to the reader.

We leave the determination of $n\left(p^{7}, 2\right)$ as an exercise to the reader. Let us just mention that the value of this function depends on the class of the prime $p \bmod 3 \cdot 5 \cdot 11 \cdot 13 \cdot 17$, and that its range is equal to $\{1,2, \ldots, 8\}$. The case $k=8$ is much simpler. We state the result without proof.

Proposition 4.2 Let $p$ be an odd prime number. Then we have:

$$
n\left(p^{8}, 2\right)=\left\{\begin{array}{lll}
6 & \text { if } & \rho_{7,31,127}(p)=(5,23,63) \\
7 & \text { if } & \rho_{7,31,127}(p)=(\neg 5,23,63),(5, \neg 23,63) \text { or }(5,23, \neg 63) \\
8 & \text { if } & \rho_{7,31,127}(p)=(\neg 5, \neg 23,63),(\neg 5,23, \neg 63) \text { or }(5, \neg 23, \neg 63) \\
9 & \text { if } \quad \rho_{7,31,127}(p)=(\neg 5, \neg 23, \neg 63) .
\end{array}\right.
$$

That was the gentle side of the story. Here comes the harder one. 


\subsection{When $k$ grows}

When $k$ grows arbitrarily, the task of determining $n\left(p^{k}, 2\right)$ for all odd primes $p$ using Proposition 2.2 becomes much more complicated, and turns out to be linked to hard problems. Let us focus on one specific factorization of $2 p^{k}$, namely $2 p^{k}=u v$ with

$$
u=p^{k-1}, v=2 p .
$$

In order to find when this factorization contributes 1 to $n\left(p^{k}, 2\right)$, we need to decide when $\operatorname{gcd}\left(p^{k-1}+1,2 p+1\right)$ is equal to 1 . Here is the key reduction.

Lemma 4.3 Let $p$ be an odd prime and let $m \in \mathbb{N}$. Then

$$
\operatorname{gcd}\left(p^{m}+1,2 p+1\right)= \begin{cases}\operatorname{gcd}\left(2^{m}+1,2 p+1\right) & \text { if } m \text { is even } \\ \operatorname{gcd}\left(2^{m}-1,2 p+1\right) & \text { if } m \text { is odd }\end{cases}
$$

Proof. As earlier, we will reduce $\operatorname{gcd}\left(x^{m}+1,2 x+1\right)$ in $\mathbb{Z}\left[2^{-1}, x\right]$ to the form $\operatorname{gcd}(f, g)$, where either $f$ or $g$ is a constant polynomial. Since $x \equiv-2^{-1} \bmod (2 x+1)$, trick $(5)$ yields

$$
\begin{aligned}
\operatorname{gcd}\left(x^{m}+1,2 x+1\right) & =\operatorname{gcd}\left((-2)^{-m}+1,2 x+1\right) \\
& =\operatorname{gcd}\left(2^{m}+(-1)^{m}, 2 x+1\right) .
\end{aligned}
$$

Substituting $x=p$ gives the stated formula.

Hence, in order to determine when $\operatorname{gcd}\left(p^{m}+1,2 p+1\right)$ equals 1 , we need to know the prime factors of $2^{m}+1$ for $m$ even, and of $2^{m}-1$ for $m$ odd. This is an ancient open problem. It is not even known at present whether there are finitely or infinitely many Fermat or Mersenne primes, i.e. primes of the form $F_{t}=2^{2^{t}}+1$ or $M_{q}=2^{q}-1$ with $t \geq 0$ and $q$ prime, respectively.

- Assume for instance that $k=2^{t}+1$ for some $t \geq 1$. Then $k-1$ is even, and thus Lemma 4.3 yields

$$
\operatorname{gcd}\left(p^{k-1}+1,2 p+1\right)=\operatorname{gcd}\left(F_{t}, 2 p+1\right) .
$$

Therefore, as long as the prime factors of the Fermat number $F_{t}$ remain unknown, we cannot determine those primes $p$ for which the gcd in (10) equals 1 , and hence write down an exact formula for $n\left(p^{k}, 2\right)$ in the spirit of Proposition 4.1. For the record, as of 2010, the prime factorization of $F_{t}$ is completely known for $t \leq 11$ only [5]. 
- Assume now that $k=q+1$ for some large prime $q$. Then $k-1=q$ is odd, and Lemma 4.3 yields

$$
\operatorname{gcd}\left(p^{k-1}+1,2 p+1\right)=\operatorname{gcd}\left(2^{q}-1,2 p+1\right) .
$$

Here again, we do not know the prime factors of $M_{q}=2^{q}-1$ in general; it may even happen that $2^{q}-1$ hits some unknown Mersenne prime. Thus, we will not know for which primes $p$ the gcd in (11) equals 1, i.e. when the specific factorization $2 p^{k}=p^{k-1} \cdot 2 p$ contributes 1 to $n\left(p^{k}, 2\right)$. For the record, the largest prime currently known is the Mersenne prime $p=2^{43,112,609}-1$, found in August 2008 [4].

The above difficulties concern the specific factorization $2 p^{k}=p^{k-1} \cdot 2 p$. However, most other ones will also lead to trouble for some exponents $k$. For instance, consider the factorization $2 p^{k}=p^{k-2} \cdot 2 p^{2}$, and let $k=2^{t+1}+2$. Then, a computation as in the proof of Lemma 4.3 yields

$$
\operatorname{gcd}\left(p^{k-2}+1,2 p^{2}+1\right)=\operatorname{gcd}\left(F_{t}, 2 p^{2}+1\right) \text {. }
$$

Once again, not knowing the prime factors of $F_{t}=2^{2^{t}}+1$ prevents us to know for which primes $p$ this ged equals 1 .

\section{Concluding remarks and open questions}

We have determined $n(g, 2)$ when $2 g-1$ is prime, for $g=2^{k}$ for all $k \geq 1$, and for $g=p^{k}$ for all odd primes $p$ and $k \leq 6$. The general case is probably out of reach. However, here are a few questions which might be more tractable, yet which we cannot answer at present.

1. Is there an explicit formula for $n\left(3^{k}, 2\right)$ as a function of $k$ ? Is it true that $n\left(3^{k}, 2\right)$ goes to infinity as $k$ does?

Here are the values of this function for $k=1,2, \ldots, 20$ :

$$
2,3,4,4,5,7,8,9,8,9,11,13,11,15,16,14,14,18,20,21 .
$$

2. Can one characterize those integers $g \geq 1$ for which $n(g, 2)=1$ ?

In special cases, we know enough to get a complete answer, for instance when $g$ is prime using Proposition 4.1, or when $g=2^{k}$ using 
Theorem 3.1. However, the general case seems to be very hard. As an appetizer, let us mention that a prime $p$ satisfies $n\left(p^{21}, 2\right)=1$ if and only if $p \equiv 8 \bmod 3 \cdot 5 \cdot 17 \cdot 257 \cdot 65537$; the smallest such prime is

$$
p=12,884,901,893 \text {. }
$$

3. Let $r \in \mathbb{N}, r \geq 1$. Does $n\left(p_{1} \cdots p_{r}, 2\right)$ attain every value $i \in\left\{1,2, \ldots, 2^{r}\right\}$ for suitable distinct primes $p_{1}, \ldots, p_{r}$, and infinitely often so?

4. Let $l \in \mathbb{N}, l \geq 1$. Does $n\left(p^{2 l-1}, 2\right)$ attain every value $i \in\{1,2, \ldots, 2 l\}$ for suitable odd primes $p$, and infinitely often so?

5. In contrast, is it true that $\min \left\{n\left(p^{2 l}, 2\right) \mid p\right.$ odd prime $\}$ goes to infinity with $l$ ?

Using the above methods, and a classical theorem of Dirichlet, it is fairly easy to show that, independently of the parity of $k$, the function $n\left(p^{k}, 2\right)$ attains its maximal value $k+1$ infinitely often.

\section{References}

[1] M. Bras-Amorós, Fibonacci-like behavior of the number of numerical semigroups of a given genus, Semigroup Forum 76 (2008) 379-384.

[2] M. Bras-Amorós, Bounds on the number of numerical semigroups of a given genus, J. Pure and Applied Algebra 213 (2009) 997-1001.

[3] R. Fröberg, C. Gottlieb, R. HäGgkvist, On numerical semigroups, Semigroup Forum 35 (1987) 63-83.

[4] GIMPS, Great Internet Mersenne Prime Search, http://www . mersenne.org/.

[5] W. Keller, Prime factors $k \cdot 2^{n}+1$ of Fermat numbers $F_{m}$ and complete factoring status, web page available at http://www.prothsearch.net/fermat.html.

[6] J.L. Ramírez Alfonsín, The Diophantine Frobenius problem, Oxford Lecture Series in Mathematics and its Applications 30, Oxford University Press, Oxford, (2005). 
[7] J.J. Sylvester, On subinvariants, i.e. semi-invariants to binary quantities of an unlimited order, Amer. J. Math. 5 (1882) 119-136.

\section{Authors addresses:}

- Shalom Eliahou ${ }^{\mathrm{a}, \mathrm{b}, \mathrm{c}}$,

${ }^{a}$ Univ Lille Nord de France, F-59000 Lille, France

${ }^{\mathrm{b}}$ ULCO, LMPA J. Liouville, B.P. 699, F-62228 Calais, France

${ }^{\mathrm{c}} \mathrm{CNRS}$, FR 2956, France

e-mail: eliahou@lmpa.univ-littoral.fr

- Jorge Ramírez Alfonsín,

Institut de Mathématiques et de Modélisation de Montpellier

Université Montpellier 2

Case Courrier 051

Place Eugène Bataillon

34095 Montpellier, France

UMR 5149 CNRS

e-mail: jramirez@math.univ-montp2.fr 